\title{
Evoked potentials recorded during routine EEG predict outcome after perinatal asphyxia
}

\section{Nevalainen, Päivi}

2017-07

Nevalainen, P , Marchi , V , Metsäranta , M , Lönnqvist , T, Toiviainen-Salo , S, Vanhatalo , S \& Lauronen , L 2017 , ' Evoked potentials recorded during routine EEG predict outcome after perinatal asphyxia ' , Clinical Neurophysiology , vol. 128 , no. 7 , pp. 1337-1343 . https://doi.org/10.1016/j.clinph

http://hdl.handle.net/10138/297841

https://doi.org/10.1016/j.clinph.2017.04.025

publishedVersion

Downloaded from Helda, University of Helsinki institutional repository.

This is an electronic reprint of the original article.

This reprint may differ from the original in pagination and typographic detail.

Please cite the original version. 


\title{
Evoked potentials recorded during routine EEG predict outcome after perinatal asphyxia
}

\author{
Päivi Nevalainen ${ }^{\mathrm{a}, 1, *}$, Viviana Marchi ${ }^{\mathrm{b}, \mathrm{c}, 1}$, Marjo Metsäranta ${ }^{\mathrm{d}}$, Tuula Lönnqvist ${ }^{\mathrm{e}}$, Sanna Toiviainen-Salo ${ }^{\mathrm{f}}$, \\ Sampsa Vanhatalo ${ }^{\mathrm{a}}$, Leena Lauronen ${ }^{\mathrm{a}}$ \\ a Department of Clinical Neurophysiology, Children's Hospital, HUS Medical, Imaging Center, University of Helsinki and Helsinki University Hospital (HUH), Helsinki, Finland \\ ${ }^{\mathrm{b}}$ Department of Developmental Neuroscience, Stella Maris Scientific Institute, IRCCS Stella Maris Foundation Pisa, Italy \\ ${ }^{c}$ Department of Clinical and Experimental Medicine, University of Pisa, Pisa, Italy \\ ${ }^{\mathrm{d}}$ Department of Neonatology, Children's Hospital, University of Helsinki and HUH, Helsinki, Finland \\ ${ }^{\mathrm{e}}$ Department of Child Neurology, Children's Hospital, University of Helsinki and HUH, Helsinki, Finland \\ ${ }^{\mathrm{f}}$ HUS Medical Imaging Center, Radiology, University of Helsinki and HUH, Helsinki, Finland
}

\section{A R T I C L E I N F O}

\section{Article history:}

Accepted 26 April 2017

Available online 11 May 2017

\section{Keywords:}

Somatosensory evoked potentials

Visual evoked potentials

Electroencephalography

Asphyxia

Newborn

Brain monitoring

\section{H I G H L I G H T S}

- EEG with simultaneous evoked potential recording is valuable in asphyxic newborns.

- Somatosensory evoked potentials recorded during routine EEG predict outcome with $98 \%$ accuracy.

- EEG complemented with SEPs is more accurate than EEG alone in outcome prediction.

\begin{abstract}
A B S T R A C T
Objective: To evaluate the added value of somatosensory (SEPs) and visual evoked potentials (VEPs) recorded simultaneously with routine EEG in early outcome prediction of newborns with hypoxicischemic encephalopathy under modern intensive care.

Methods: We simultaneously recorded multichannel EEG, median nerve SEPs, and flash VEPs during the first few postnatal days in 50 term newborns with hypoxic-ischemic encephalopathy. EEG background was scored into five grades and the worst two grades were considered to indicate poor cerebral recovery. Evoked potentials were classified as absent or present. Clinical outcome was determined from the medical records at a median age of 21 months. Unfavorable outcome included cerebral palsy, severe mental retardation, severe epilepsy, or death.

Results: The accuracy of outcome prediction was $98 \%$ with SEPs compared to $90 \%$ with EEG. EEG alone always predicted unfavorable outcome when it was inactive $(n=9)$, and favorable outcome when it was normal or only mildly abnormal $(n=17)$. However, newborns with moderate or severe EEG background abnormality could have either favorable or unfavorable outcome, which was correctly predicted by SEP in all but one newborn (accuracy in this subgroup 96\%). Absent VEPs were always associated with an inactive EEG, and an unfavorable outcome. However, presence of VEPs did not guarantee a favorable outcome.

Conclusions: SEPs accurately predict clinical outcomes in newborns with hypoxic-ischemic encephalopathy and improve the EEG-based prediction particularly in those newborns with severely or moderately abnormal EEG findings.

Significance: SEPs should be added to routine EEG recordings for early bedside assessment of newborns with hypoxic-ischemic encephalopathy.

(c) 2017 International Federation of Clinical Neurophysiology. Published by Elsevier Ireland Ltd. All rights
\end{abstract} reserved.

\footnotetext{
Abbreviations: aEEG, amplitude-integrated electroencephalography; BE, base excess; CP, cerebral palsy; EEG, electroencephalography; EP, evoked potential; HIE, hypoxic-

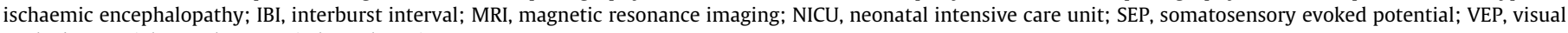
evoked potential; TH, therapeutic hypothermia.

* Corresponding author at: Department of Clinical Neurophysiology, Childrens' Castle, Helsinki University Hospital, P.O. Box 280, 00029 HUS, Finland.

E-mail address: paivi.nevalainen@hus.fi (P. Nevalainen).

1 These authors contribute equally to the manuscript.
} 


\section{Introduction}

Hypoxic-ischaemic encephalopathy (HIE) due to perinatal asphyxia occurs in approximately 2,5 per 1000 live full-term births (Graham et al., 2008), and is one of the leading causes of neonatal deaths and severe neurodevelopmental compromise (Lai and Yang, 2011). Recent data show that even when treated with therapeutic hypothermia (TH), $40-50 \%$ of newborns with moderate-tosevere HIE will die or develop with severe handicap (Tagin et al., 2012).

Early recovery of the electroencephalography (EEG) background or amplitude-integrated EEG (aEEG) trend has, within the last decade, become a routine bedside method for early prognostication (Hellström-Westas et al., 1995; Toet et al., 1999; Murray et al., 2009). However, TH delays the aEEG recovery beyond the first 36-48 h, limiting its utility in early outcome prediction (Hallberg et al., 2010; Thoresen et al., 2010; Massaro et al., 2012; Csekő et al., 2013; Bonifacio et al., 2015). In terms of early prognostication, the newborns with moderate HIE are the most challenging (Gunn et al., 2008). This very same population may, however, gain the most from therapeutic interventions (Tagin et al., 2012). Hence, it is of high priority to improve the accuracy of outcome prediction at bedside to support individualized treatment decisions.

In addition to EEG, other neurophysiological methods, particularly somatosensory (SEPs) (Majnemer et al., 1990; Taylor et al., 1992; Eken et al., 1995; Scalais et al., 1998; Suppiej et al., 2010; Swarte et al., 2012; Kontio et al., 2013) and visual evoked potentials (VEPs) (Whyte et al., 1986; Muttitt et al., 1991; Taylor et al., 1992; Eken et al., 1995; Scalais et al., 1998; Suppiej et al., 2010) are known to predict clinical outcome after perinatal asphyxia. In the present paper, we set out to study whether recording SEPs and VEPs simultaneously with routine EEG could provide additional value in the early outcome prediction in term newborns with HIE, and whether their predictive yield is affected by $\mathrm{TH}$, an essential part of modern neonatal intensive care.

\section{Patients and methods}

\subsection{Patients (Table 1, Supplementary Table S1)}

The study included 50 newborns (born between $36+7$ and 42 +2 gestational weeks; 23 females) treated for asphyxia/HIE at the tertiary level neonatal intensive care unit (NICU) of the Helsinki University Hospital during a four-year period (January 2011 December 2014). Initially, we identified all asphyxiated/HIE newborns within the study period that underwent an EEG. Asphyxia was defined by the following criteria: $\mathrm{pH}<7.10, \mathrm{BE} \leq-10 \mathrm{mmol}$, 5 -min Apgar score $\leq 6$, or primary mechanical ventilation and some other indication of asphyxia (e.g. abnormal cardiotocography, decreased fetal movements, or meconium in the amniotic fluid). We then excluded newborns with gestational age $<36$ weeks and those with diagnosed or suspected genetic abnormalities, inborn errors of metabolism, or major malformations. Only the earliest EEG of those newborns with several recordings was included. All of the final 50 EEG recordings included simultaneous SEPs and VEPs (in accordance with our in-house routine clinical neonatal EEG protocol). All neurophysiological recordings were performed for clinical indications. Of these 50 newborns, 28 received wholebody therapeutic hypothermia (target temperature 33-34 degrees) for $72 \mathrm{~h}$ as part of their treatment strategy and 15 of the 28 were still under hypothermia during the EEG and EP recording. Cooling was initiated according to the criteria of the TOBY study (Azzopardi et al., 2008) with the exception that in certain situation cooling was initiated without a preceding aEEG trace (e.g. if aEEG was not available).
The Ethics Committee for Pediatrics, Adolescent medicine, and Psychiatry, Hospital District of Helsinki and Uusimaa, approved the study protocol. Parental consent form was received for the use of the photograph in Fig. 1A.

\subsection{Decisions on withdrawal of treatment}

Decisions to withdraw intensive care were based on a combination of poor clinical condition including severe HIE, poor EEG, and severe MRI findings. Decisions were made after discussions with parents.

\subsection{EEG, SEP, and VEP recording (Fig. $1 A$ and $B$ )}

The EEG and EPs were recorded between $15 \mathrm{~h}$ and 10 days postnatally, following our in-house developed clinical routines (Nevalainen et al., 2015). We collected the EEG and SEP signals at $2000 \mathrm{~Hz}$ using the NicoletOne EEG system (Cardinal Healthcare/ Natus, USA; acquisition bandwidth $0.053-500 \mathrm{~Hz}$ ), $\mathrm{Cz}$ reference, and 21 channel EEG caps (sintered $\mathrm{Ag} / \mathrm{AgCl}$ electrodes; Waveguard, ANT-Neuro, Germany). An additional electrode was placed over the C7 vertebra to record the cervical SEP. We stimulated each median nerve at the wrist at $0.5(n=7)$ or $1 \mathrm{~Hz}(n=43)$ rates using two disk electrodes and a battery powered portable electrical peripheral nerve stimulator (Micromed Energy Light stimulator; Micromed, Italy) and pulse width of $0.2 \mathrm{~ms}$. If standard stimulation was not possible due to intravenous lines, we stimulated the median nerve at the palm $(n=7)$ or elbow $(n=1)$. One newborn only underwent unilateral stimulation because no suitable stimulation site was accessible on one side. The stimulation current was individually adjusted to just above the motor threshold. VEP stimulations were delivered using a stroboscopic flash at 1 or $2 \mathrm{~Hz}$. We aimed at recording all the evoked potentials during sleep (or quietness in case normal vigilance states could not be identified). However, in each EP condition (right hand SEP, left hand SEP, VEP) three newborns did not fall asleep within the recording time slot or woke up during the recording and were, thus, recorded while awake.

\subsection{EEG background grading}

Two EEG experts (PN and VM) blinded to the clinical information scored all EEGs independently using previously described criteria (Murray et al., 2009). In case of disagreement, a third EEG expert (LL) scored the given EEG, and the final score was reached by consensus. EEG grade 4 indicated inactive trace (background activity $<10 \mu \mathrm{V}$ or severe discontinuity with interburst interval (IBI) $>60 \mathrm{~s}$ ), grade 3 severe abnormality (discontinuous activity with IBI 10-60 s, severe attenuation of background patterns, no sleep-wake cycle), grade 2 moderate abnormality (discontinuous activity with IBI $<10 \mathrm{~s}$, or clear asymmetry or asynchrony), grade 1 mild abnormality (continuous activity with slightly abnormal activity: e.g., mild asymmetry, or mild voltage depression), and grade 0 normal EEG.

\subsection{Analysis of evoked potentials}

We averaged the EPs offline in BESA ${ }^{\circledR}$ software (BESA GmbH, Germany) for epochs from -100 to $800 \mathrm{~ms}$ relative to stimulus onset without further filtering (Fig. 1C). Author PN visually evaluated all EPs using bipolar montages and electrical field maps (Fig. 1D-E). Cervical SEP was identified in the cervical electrode (C7, referenced to Fz) between 8 and $20 \mathrm{~ms}$. Cortical SEP was observed as a salient response beginning within $100 \mathrm{~ms}$ from the stimulation in the contralateral centroparietal area coupled with a topographic pattern of electrical field that indicated a source at the contralateral primary somatosensory cortex i.e. a parietal 
A

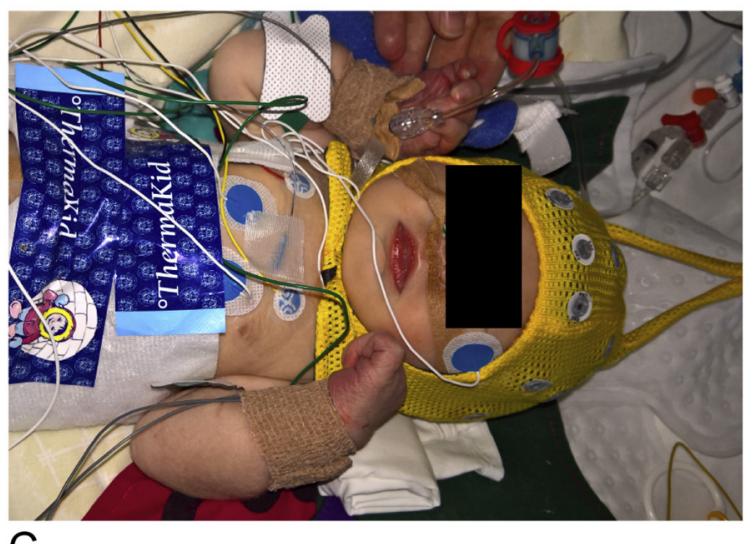

$\mathrm{B}$

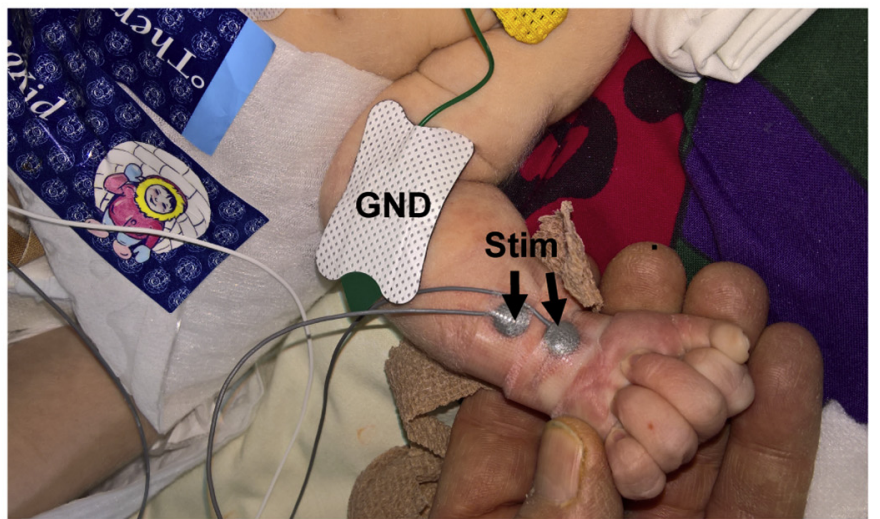

C
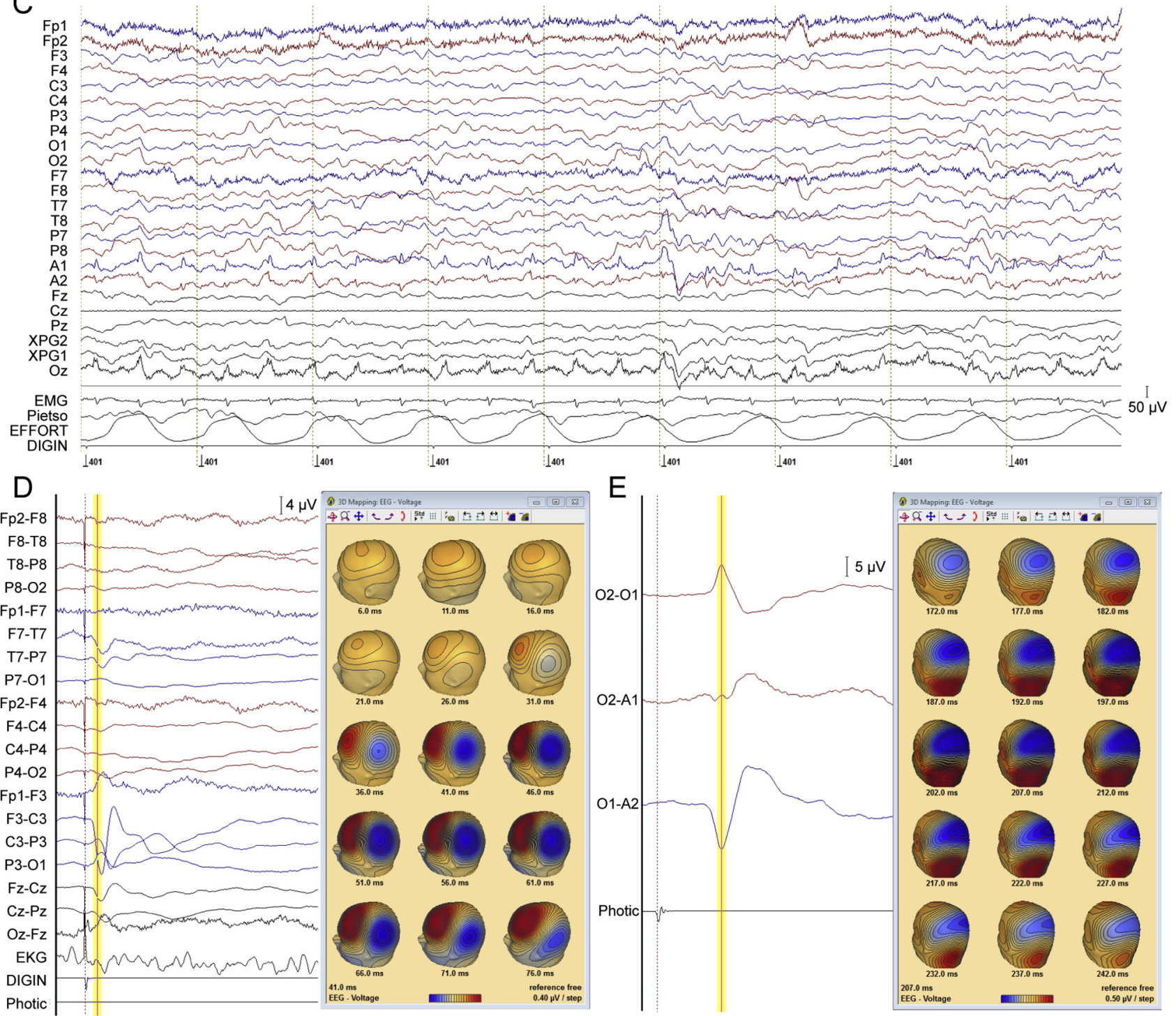

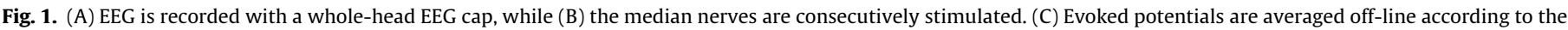

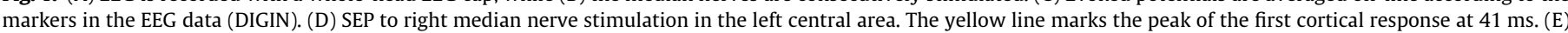
VEP in the occipital area. The yellow line marks the peak of the response at $207 \mathrm{~ms}$.

negativity paired with a frontal positivity. Cortical VEPs were observed in the occipital area with onset $<300 \mathrm{~ms}$. Cortical SEPs were classified as bilaterally absent, unilaterally absent, or bilater- ally present, whereas VEPs were classified as absent or present. The classification agreement rate with the original clinical EP report was $100 \%$. 


\subsection{Magnetic resonance imaging (MRI)}

Of the 50 newborns, 46 underwent brain MRI (1.5Tesla scanner, Philips Intera Achieva, Philips Medical Systems, Best, The Netherlands) with a clinical imaging protocol including T1-weighted axial, T2-weighted axial and coronal, diffusion weighted axial, and $\mathrm{T} 2$ gradient echo axial images. An experienced pediatric neuroradiologist (author STS) classified the MRIs into six categories according to previously published criteria (Shankaran et al., 2012) (see Supplementary Table S1 for details).

\subsection{Outcome}

An experienced neonatologist (author $\mathrm{MM}$ ) determined clinical outcomes by retrospective review of the medical records using the following predifined, outcome categories (see also Iyer et al., 2014): normal, mild abnormality (including mild speech, motor, or cognitive delay), moderate abnormality (including moderate dyskinetic or hemiplegic $\mathrm{CP}$ ) severe abnormality (including tetraplegic and/or severe dyskinetic $\mathrm{CP}$, severe mental retardation, and severe epilepsy), or death. Normal and mild abnormality were considered as favorable and moderate/severe abnormality and death as unfavorable outcomes. The outcome scoring was primarily based on an evaluation from the routine follow-up visit to a (neuro)pediatrician between 18 and 30 months age. When this was not available (17 patients) we used the latest available visit between 3 and 16 months age (see Supplementary Table S1 for details).

\subsection{Statistics}

We calculated the accuracy, sensitivity, specificity, and positive and negative predictive values, including corresponding 95\% confidence intervals, for the predefined, dichotomic neurophysiological results: Poor EEG was taken as grade 3-4, poor SEP as absent response on at least one hemisphere, and poor VEP as an absent response.

\section{Results}

EEG grade and presence/absence of EPs were all predictive of outcome (Fig. 2). The prediction accuracy was highest with SEPs (98\%), followed by EEG (90\%), and lowest with VEPs (84\%). Specificity, sensitivity, and positive and negative predictive values for each method and their combinations are shown in Table 2. In the clinically most challenging group of 24 newborns with moderateto-severe EEG background abnormality (grade 2 and 3), the accuracy of outcome prediction with EEG was $79 \%$, whereas accuracy of prediction with SEP was $96 \%$.

EEG and SEP results with respect to postnatal age during the recording are shown in Fig. 3. For those recordings performed within the first $48 \mathrm{~h}$, the prediction accuracy of SEP was $100 \%$ and that of EEG 93\%. In the subgroup of newborns that received therepeutic hypothermia the prediction accuracy of SEPs was $100 \%$, and that of EEG $96 \%$.

Individual EEG and EP results, together with the corresponding clinical details and MRI scores, are presented in Supplementary Table S1. Of the 50 newborns, EEG showed grade 4 background abnormality in nine newborns, of whom SEPs were bilaterally absent in eight and unilaterally absent in one. All nine had a poor outcome (six died in the neonatal period, three developed severe disability and one of them died at 5-months of age). EEG grade 3 was observed in eight newborns. SEPs were bilaterally absent in four and unilaterally absent in one of them, and all five had an unfavorable outcome (one died and four developed with moderate

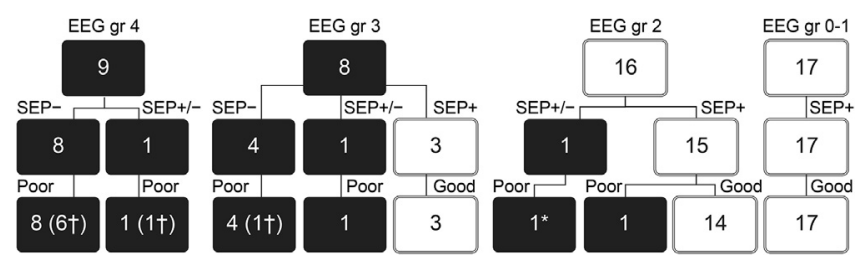

Fig. 2. Flow diagrams: association between EEG, SEP, and outcome. Black squares mark poor test result/unfavorable outcome, and white squares mark good test result/favorable outcome. Numbers within squares mark the number of newborns. SEP $-=$ SEP bilaterally absent, SEP $+1-=$ SEP unilaterally absent, SEP $+=$ SEP bilater ally present, $\dagger=$ number of deaths, ${ }^{*}=$ hemiplegic cerebral palsy.

Table 1

Baseline characteristics of the study population.

\begin{tabular}{ll}
\hline Baseline characteristic & $N=50$ \\
\hline Females & $23(46 \%)$ \\
Received TH & $28(56 \%)$ \\
Under TH during EEG-EP & $15(30 \%)$ \\
GA & $40[1,5]$ weeks \\
Postnatal age at EEG-EP & $45\{66\} \mathrm{h}$ \\
Birth weight & $3330[630] \mathrm{g}$ \\
BE & $-13,5[5,9]$ \\
pH & $7,0[0,2]$ \\
Apgar 1 min & $1\{1\}$ \\
Apgar 5 min & $3\{4\}$ \\
Apgar 10 min & $5\{3\}$ \\
\hline
\end{tabular}

Data shown as $n(\%)$, mean [SD], or median $\{\mathrm{IQR}\}$. TH $=$ therapeutic hypothermia, $\mathrm{GA}=$ gestational age, $\mathrm{EP}=$ evoked potential, $\mathrm{BE}=$ base excess.

or severe disability). On the contrary, three newborns with EEG grade 3 but present SEPs had a favorable outcome. EEG grade 2 was observed in 16 newborns, one of which had unilaterally absent SEP and an unfavorable outcome (moderate disability, hemiplegia). Of the remaining 15 newborns with SEPs present bilaterally, fourteen had a favorable outcome, but one (not receiving hypothermia) developed severe disability. This newborn had the largest asymmetry in SEP onset latency ( $8 \mathrm{~ms}$ vs other newborns' median $1 \mathrm{~ms}$, range 0-6 ms), which was, however, not part of the predefined SEP criteria. EEG was normal or only mildly abnormal (grade 0 or 1) in the remaining 17 newborns. All of them had bilateral SEPs, and a favorable outcome. VEPs were absent in eight of the nine newborns with grade $4 \mathrm{EEG}$, and present in all the other 42 newborns.

\section{Discussion}

Our data show that assessment of evoked potentials simultaneously with a routine EEG provides a reliable bedside tool for early outcome prediction in term newborns with HIE. In line with previous literature (Pressler et al., 2001; Murray et al., 2009; Hamelin et al., 2011), normal or mildly abnormal EEG background was invariably associated with a favorable outcome, while an inactive EEG predicted an unfavorable outcome. Newborns with moderate-to-severe EEG background abnormality (EEG grade 23 ) could have either outcome, which was, however, reliably predicted by the simultaneous SEP. Absent VEPs were only observed in the newborns whose unfavorable outcome was already predicted correctly by the inactive EEG.

\subsection{Challenges in early outcome prediction after perinatal asphyxia}

Early outcome prediction in asphyxiated newborns is essential for guiding individual treatment decisions, but it is complicated by the combination of rapidly changing neurological state during 
Table 2

Predictive values of poor EEG, SEPs, and VEPs and their combinations for an unfavorable outcome.

\begin{tabular}{|c|c|c|c|c|}
\hline Method(s) & Specificity & Sensitivity & PPV & NPV \\
\hline EEG & $91(76-98)$ & $88(62-98)$ & $82(57-96)$ & $94(80-99)$ \\
\hline SEP & $100(90-100)$ & $94(70-100)$ & $100(78-100)$ & $97(85-100)$ \\
\hline VEP & $100(90-100)$ & $50(25-75)$ & $100(63-100)$ & $81(66-91)$ \\
\hline EEG and SEP & $100(90-100)$ & $88(62-98)$ & $100(77-100)$ & $94(81-99)$ \\
\hline EEG and VEP & $100(90-100)$ & $50(25-75)$ & $100(63-100)$ & $81(66-91)$ \\
\hline EEG and SEP and VEP & $100(90-100)$ & $50(25-75)$ & $100(63-100)$ & $81(66-91)$ \\
\hline EEG or SEP & $91(76-98)$ & $94(70-100)$ & $83(59-96)$ & $97(84-100)$ \\
\hline EEG or VEP & $91(76-98)$ & $88(62-98)$ & $82(57-96)$ & $94(80-99)$ \\
\hline EEG or SEP or VEP & $91(76-98)$ & $94(70-100)$ & $83(59-96)$ & $97(84-100)$ \\
\hline
\end{tabular}

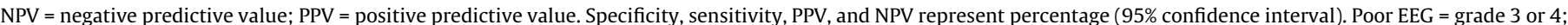
poor SEPs = SEP absent from at least one hemisphere; poor VEPs = VEPs absent.

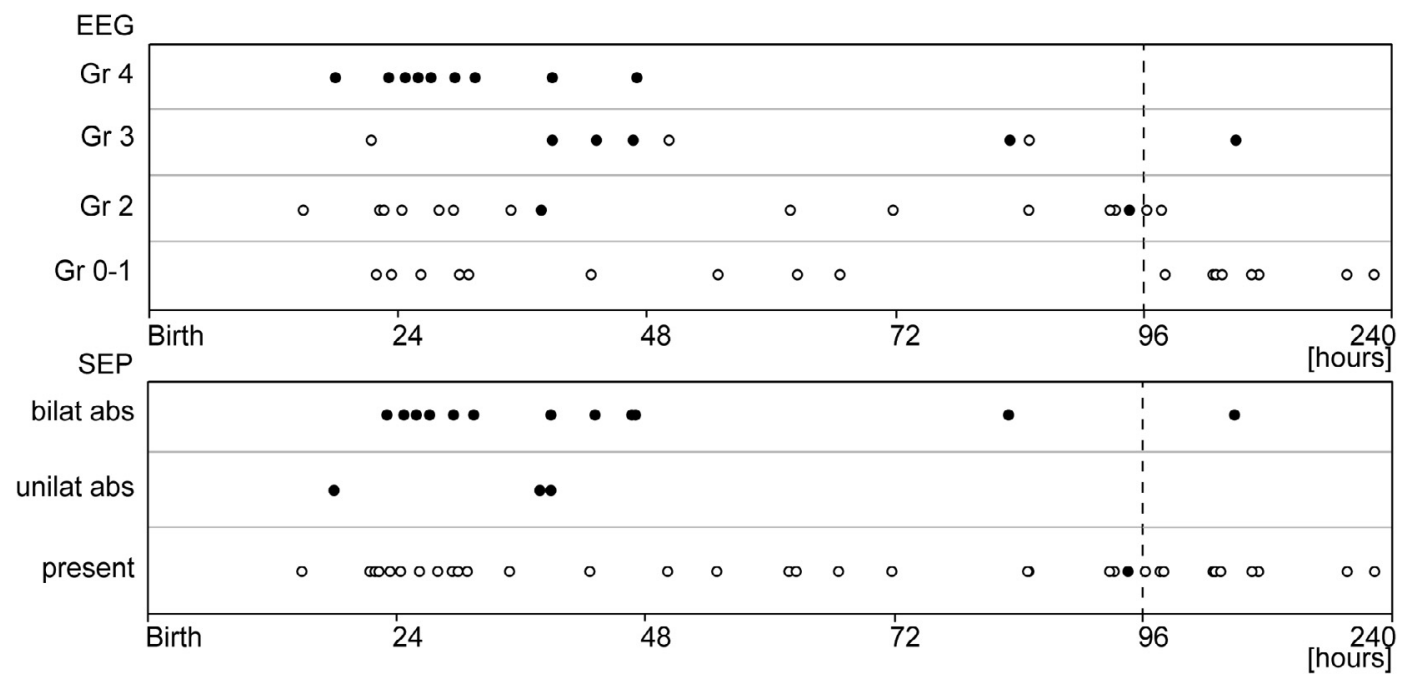

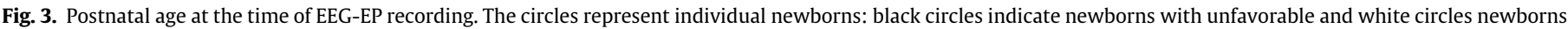
with favorable outcome. The dashed line marks a change in the time scale.

recovery from HIE, possible secondary injury, and the hypothermia treatment. Several parameters measured at birth (e.g. Apgar score, and severity of acidaemia) are associated with outcomes but, individually, they tend to have poor predictive accuracy (Merchant and Azzopardi, 2015). The clinically determined grade of HIE (Sarnat and Sarnat, 1976) by itself predicts later outcome, but newborns with moderate HIE present a clinical challenge as their outcomes vary considerably (Gunn et al., 2008). Neonatal MRI is highly predictive of outcome, both in normothermic (Rutherford et al., 1998) and TH-treated HIE newborns (Rutherford et al., 2010; Cheong et al., 2012), but it is not available at bedside, and the severity of injury may in some cases be underestimated during the first two days after birth (Barkovich et al., 2006).

\subsection{EEG in outcome prediction}

EEG is widely used for early outcome prediction in asphyxiated term newborns. Our present results support the general consensus that a normal or mildly abnormal EEG background during the first postnatal days is predictive of a favorable outcome (Pressler et al., 2001; Murray et al., 2009; Hamelin et al., 2011). On the contrary, a severely abnormal EEG after hypoxic-ischemic insult only predicts a poor outcome if it fails to recover within the first day (Pressler et al., 2001; Murray et al., 2009). Observing this recovery, has been the basis for aEEG -based outcome prediction until the introduction of $\mathrm{TH}$, which turned out to significantly delay the aEEG recovery during the first days of life (Hallberg et al., 2010; Thoresen et al., 2010; Massaro et al., 2012; Csekő et al., 2013; Bonifacio et al., 2015). Our work, together with prior studies (Pressler et al., 2001; Murray et al., 2009; Hamelin et al., 2011) that have evaluated the grade of EEG background abnormality in more detail, show that an inactive or extremely discontinuous (IBI > $60 \mathrm{~s}$ ) EEG on the second postnatal day or later invariably predicts unfavorable outcome, whereas a clearly, but not as severely, discontinuous pattern (IBI 10-60 s) may lead to either unfavorable or favorable outcome (Murray et al., 2009). This suggests a need for improved methods to extract signatures of brain viability from among the burst suppression backgrounds (Iyer et al., 2014). Our present work demonstrates that SEPs may aid in outcome prediction in this particular group of infants.

\subsection{Evoked potentials in outcome prediction}

Evoked potentials assess the functional integrity of the given sensory system including key pathways in the deep brain structures (e.g. thalami and brainstem), which are known to be vulnerable to perinatal asphyxia (Gunn and Bennet, 2009). Studies in non-human primates suggest that compared to isoelectric EEG, cortical SEPs would be lost at slightly lower cerebral blood flow values, and thus at a level of ischemia nearer to the true critical level for neuronal damage (Dong et al., 1986). Prior studies in normothermic HIE newborns have repeatedly shown that bilaterally absent SEPs (Eken et al., 1995; Scalais et al., 1998; Suppiej et al., 2010; Swarte et al., 2012) and VEPs (Whyte et al., 1986; Muttitt et al., 1991; Taylor et al., 1992; Eken et al., 1995; Scalais et al., 1998; Suppiej et al., 2010) are highly predictive of an unfavorable 
outcome, and bilaterally present SEPs strongly predictive of a favorable outcome (Taylor et al., 1992; Eken et al., 1995; Scalais et al., 1998; Suppiej et al., 2010; Swarte et al., 2012), whereas normal VEPs have lower prognostic value (Taylor et al., 1992; Suppiej et al., 2010) (but see also Whyte et al., 1986; Muttitt et al., 1991; Scalais et al., 1998). Reports on the predictive yield of unilaterally absent SEPs or delays in SEP latencies have been more variable (Eken et al., 1995; Suppiej et al., 2010; Swarte et al., 2012). Previous studies have, however, used a limited number of recording electrodes, whereas we used multichannel SEP assessment including offline averaging and montaging for an improved SEP identification. We believe that these methodological advances improved the accuracy enough to show that also all newborns with unilaterally absent SEPs had an unfavorable outcome. Importantly, since our method - simultaneous EEG and SEP recording - allows interpreting SEPs with the knowledge of spontaneous cortical (EEG) activity, we were able to show that SEPs could distinguish the newborns with favorable and unfavorable outcome in the challenging group of 8 newborns with discontinuous EEG background (grade 3 ). Bilaterally present SEPs predicted favorable outcome with the exception of only one newborn. Our posthoc analysis suggested that this newborn had an exceptionally large asymmetry $(8 \mathrm{~ms})$ in cortical SEP onset latency, which was not part of our predefined SEP criteria. Future studies with other datasets are needed to define whether onset latency asymmetry could be a useful criterion to perfect the SEP-based prognostication.

A key advantage of SEPs compared to EEG is its resistance to therapeutic levels of hypothermia, which is clearly documented both in experimental animals (Madhok et al., 2012) and in adults treated with TH after cardiac arrest (Sandroni et al., 2014). Similarly, in the present study, TH did not lower the predictive yield of SEPs as the only newborn with bilaterally present SEP but poor outcome did not undergo TH. On the contrary, a recent study in asphyxiated newborns reported only $36 \%$ PPV for bilaterally absent SEPs in predicting moderate-to-severe disability (Garfinkle et al., 2015). We suggest that several methodological differences would readily explain the markedly higher accuracies in our work: The previous study (i) recorded patients during the second week of life only; (ii) used higher stimulation frequency that is known to deteriorate SEPs in young infants (Araki et al., 1999); (iii) used only two recording electrodes as opposed to our 21 electrodes, which according to our experience will lead to ignoring at least some of the responses. Indeed, this comparison illustrates the need to reconsider neonatal SEP protocols so that they take into account the highly focal nature of neonatal scalp potentials (Odabaee et al., 2013) (i.e. more electrodes needed), and the sensitivity to stimulation frequency (Araki et al., 1999).

\subsection{Limitations of the study}

Our work was retrospective and hence we could not assess the genuine clinical impact of our EEG-EP results on actual treatment decisions. We acknowledge, that together with other clinical information and neuroimaging, EEG and to a lesser extent EPs were used in clinical decision-making concerning redirection of care, which introduces the possibility for a self-fulfilling prophecy. However, all newborns that died after redirection of care $(n=5)$ had clinically severe HIE (grade 3 ) and severe structural brain injury on MRI (grade 2B or 3, Supplementary Table S1), which are both strong independent predictors of a poor prognosis. Thus, it is likely that the outcome had been poor, even if the intensive care had been continued. Another limitation is the timing of the EEG-EP recording, which was not standardized but instead determined by the clinical question, preventing us from determining the best timing for the SEP assessment. An earlier timing of the EEG-EP recording should, however, be explored in the future to confirm a previous SEP study showing that false positive SEPs (i.e. absent SEPs associated with favorable outcome) may not be a true risk in asphyxiated newborns (Eken et al., 1995). Finally, as the follow-ups were organized according to clinical need, also their timing varied. We do, however, suggest that the follow-up information is sufficient for determining the robust outcome categories of the present study i.e. favorable vs. unfavorable.

\section{Conclusions}

This is the first study in asphyxiated newborns combining the information from simultaneously recorded EEG and EPs, which allows evaluation of EPs in the context of the brain state, particularly the spontaneous EEG background and/or sleep stage. It is conceivable that, for instance, the clinical inference of a unilaterally absent SEP together with only moderately abnormal EEG background (i.e. focal or limited brain injury) is fully different from observing unilaterally absent SEP together with an inactive EEG and absent VEP (widespread, severe injury). Hence, our results as a whole are advocating multimodal recording rather than replacement of EEG with evoked potentials.

Recording evoked potentials as a part of routine EEG is feasible, cheap, saves time, and causes minimal extra discomfort to the sick newborn. Combination of EEG and SEPs accurately predicts outcome in HIE newborns during the first days of life regardless of therapeutic hypothermia, gives a more comprehensive measure of brain function than the methods do alone (or when performed separately), and holds, thus, promise for clinical use in the future.

\section{Acknowledgements}

Our sincere thanks go to the technicians and nurses of the Department of Clinical Neurophysiology in Children's Hospital for conducting the EEG-EP measurements as well as Lotta Lauronen and Marita Suni for their help with data management. Finally, we thank the personnel of the Neonatal Intensive Care Unit at the Children's Hospital of Helsinki University Hospital for seamless co-operation. This work was supported by the Arvo and Lea Ylppö Foundation, Helsinki University Hospital Funds [Y920016024], Academy of Finland [253130], Juselius Foundation, and Foundation for Pediatric Research. The sponsors were not involved in the collection, analysis and interpretation of data or in the writing of the manuscript.

Conflict of interest: The authors have no conflicts of interest relevant to this article to disclose.

\section{Appendix A. Supplementary material}

Supplementary data associated with this article can be found, in the online version, at http://dx.doi.org/10.1016/j.clinph.2017.04. 025.

\section{References}

Araki A, Takada A, Yasuhara A, Kobayashi Y. The effects of stimulus rates on the amplitude of median nerve somatosensory evoked potentials: the developmental change. Brain Dev 1999;21(2):118-21.

Azzopardi D, Brocklehurst P, Edwards D, Halliday H, Levene M, Thoresen M Whitelaw A. TOBY Study Group. The TOBY Study. Whole body hypothermia for the treatment of perinatal asphyxial encephalopathy: a randomised controlled trial. BMC Pediatr 2008:30(8):17.

Barkovich AJ, Miller SP, Bartha A, Newton N, Hamrick SEG, Mukherjee P, et al. MR imaging, MR spectroscopy, and diffusion tensor imaging of sequential studies in neonates with encephalopathy. AJNR Am J Neuroradiol 2006;27:533-47.

Bonifacio SL, deVries LS, Groenendaal F. Impact of hypothermia on predictors of poor outcome: How do we decide to redirect care? Semin Fetal Neonatal Med 2015;20(2):122-7. 
Cheong JLY, Coleman L, Hunt RW, Lee KJ, Doyle LW, Inder TE, et al. Prognostic utility of magnetic resonance imaging in neonatal hypoxic-ischemic encephalopathy: Substudy of a randomized trial. Arch Pediatr Adolesc Med 2012;166(7):634-40.

Csekő AJ, Bangó M, Lakatos P, Kárdási J, Pusztai L, Szabó M. Accuracy of amplitudeintegrated electroencephalography in the prediction of neurodevelopmental outcome in asphyxiated infants receiving hypothermia treatment. Acta Paediatr 2013;102(7):707-11.

Dong WK, Bledsoe SW, Chadwick HS, Shaw C-M, Hornbein TF. Electrical correlates of brain injury resulting from severe hypotension and hemodilution in monkeys. Anesthesiology 1986;65:617-25.

Eken P, Toet MC, Groenendaal F, de Vries LS. Predictive value of early neuroimaging, pulsed Doppler and neurophysiology in full term infants with hypoxic-ischemic encephalopathy. Arch Dis Child 1995;73:F75-80.

Garfinkle J, Sant'Anna GM, Rosenblatt B, Majnemer A, Wintermark P, Shevell MI. Somatosensory evoked potentials in neonates with hypoxic-ischemic encephalopathy treated with hypothermia. Eur J Paediatr Neurol 2015;19 (4):423-8.

Graham EM, Ruis KA, Hartman AL, Northington FJ, Fox HE. A systematic review of the role of intrapartum hypoxia-ischemia in the causation of neonatal encephalopathy. Am J Obstet Gynecol 2008;199(6):587-95.

Gunn AJ, Bennet L. Fetal hypoxia insults and patterns of brain injury: insights from animal models. Clin Perinatol 2009;36(3):579-93.

Gunn AJ, Wyatt JS, Whitelaw A, Barks J, Azzopardi D, Ballard R, et al. Therapeutic hypothermia changes the prognostic value of clinical evaluation of neonatal encephalopathy. J Pediatr 2008;152:55-8.

Hallberg B, Grossmann K, Bartocci M, Blennow M. The prognostic value of early aEEG in asphyxiated infants undergoing systemic hypothermia treatment. Acta Paediatr 2010;99(4):531-6.

Hamelin S, Delnard N, Cneude F, Debillon T, Vercueil L. Influence of hypothermia on the prognostic value of early EEG in full-term neonates with hypoxic ischemic encephalopathy. Neurophysiol Clin 2011;41(1):19-27.

Hellström-Westas L, Rosen I, Svenningsen NW. Predictive value of early continuous amplitude integrated EEG recording on outcome after severe birth asphyxia in full term infants. Arch Dis Child 1995;72:F34-8.

Iyer KK, Roberts JA, Metsäranta M, Finnigan S, Breakspear M, Vanhatalo S. Novel features of early burst suppression predict outcome after birth asphyxia. Ann Clin Transl Neurol 2014;1(3):209-14.

Kontio T, Toet MC, Hellström-Westas L, van Handel M, Groenendaal F, Stjerna S, et al. Early neurophysiology and MRI in predicting neurological outcome at 910 years after birth asphyxia. Clin Neurophysiol 2013;124(6):1089-94.

Lai M-C, Yang S-N. Perinatal hypoxic-ischemic encephalopathy. J Biomed Biotechnol 2011;2011:1-6.

Madhok J, Wu D, Xiong W, Geocadin RG, Jia X. Hypothermia amplifies somatosensory-evoked potentials in uninjured rats. J Neurosurg Anesthesiol 2012;24(3):197-202.

Majnemer A, Rosenblatt B, Riley PS. Prognostic significance of multimodality evoked response testing in high-risk newborns. Pediatr Neurol 1990;6 (6):367-74.

Massaro AN, Tsuchida T, Kadom N, El-Dib M, Glass P, Baumgart S, et al. AEEG evolution during therapeutic hypothermia and prediction of NICU outcome in encephalopathic neonates. Neonatology 2012;102(3):197-202.

Merchant N, Azzopardi D. Early predictors of outcome in infants treated with hypothermia for hypoxic-ischaemic encephalopathy. Dev Med Child Neurol 2015;57:8-16.
Murray DM, Boylan GB, Ryan CA, Connolly S. Early EEG findings in hypoxic-ischemic encephalopathy predict outcomes at 2 years. Pediatrics 2009;124(3):e459-67.

Muttitt SC, Taylor MJ, Kobayashi JS, MacMillan L, Whyte HE. Serial visual evoked potentials and outcome in term birth asphyxia. Pediatr Neurol 1991;7 (2):86-90.

Nevalainen P, Rahkonen P, Pihko E, Lano A, Vanhatalo S, Andersson S, et al. Evaluation of somatosensory cortical processing in extremely preterm infants at term with MEG and EEG. Clin Neurophysiol 2015;126:275-83.

Odabaee M, Freeman WJ, Colditz PB, Ramon C, Vanhatalo S. Spatial patterning of the neonatal EEG suggests a need for a high number of electrodes. Neuroimage 2013;68:229-35.

Pressler RM, Boylan GB, Morton M, Binnie CD, Rennie JM. Early serial EEG in hypoxic ischaemic encephalopathy. Clin Neurophysiol 2001;112(1):31-7.

Rutherford MA, Pennock JM, Counsell SJ, Mercuri E, Cowan FM, Dubowitz LM, et al. Abnormal magnetic resonance signal in the internal capsule predicts poor neurodevelopmental outcome in infants with hypoxic-ischemic encephalopathy. Pediatrics 1998;102(2):323-8.

Rutherford M, Ramenghi LA, Edwards AD, Brocklehurst P, Halliday H, Levene M, et al. Assessment of brain tissue injury after moderate hypothermia in neonates with hypoxic-ischaemic encephalopathy: a nested substudy of a randomised controlled trial. Lancet Neurol 2010;9(1):39-45.

Sandroni C, Cariou A, Cavallaro F, Cronberg T, Friberg H, Hoedemaekers C, et al. Prognostication in comatose survivors of cardiac arrest: An advisory statement from the European Resuscitation Council and the European Society of Intensive Care Medicine. Intensive Care Med 2014;40:1816-31.

Sarnat H, Sarnat M. Neonatal encephalopathy following fetal distress. A clinical and electroencephalographic study. Arch Neurol 1976;33:696-705.

Scalais E, François-Adant A, Nuttin C, Bachy A, Guérit JM. Multimodality evoked potentials as a prognostic tool in term asphyxiated newborns. Electroenceph Clin Neurophysiol 1998;108:199-207.

Shankaran S, Barnes PD, Hintz SR, Laptook AR, Zaterka-Baxter KM, McDonald SA, et al. Brain injury following trial of hypothermia for neonatal hypoxic-ischaemic encephalopahty. Arch Dis Child Fetal neonatal Ed 2012;97(6):F398-404.

Suppiej A, Cappellari A, Franzoi M, Traverso A, Ermani M, Zanardo V. Bilateral loss of cortical somatosensory evoked potential at birth predicts cerebral palsy in term and near-term newborns. Early Hum Dev 2010;86(2):93-8.

Swarte RMC, Cherian PJ, Lequin M, Visser GH, Govaert P. Somatosensory evoked potentials are of additional prognostic value in certain patterns of brain injury in term birth asphyxia. Clin Neurophysiol 2012;123(8):1631-8.

Tagin MA, Woolcott CG, Vincer MJ, Whyte RK, Stinson DA. Hypothermia for neonatal hypoxic ischemic encephalopathy. Arch Pediatr Adolesc Med 2012;166(6):558-66.

Taylor MJ, Murphy WJ, Whyte HE. Prognostic reliability of somatosensory and visual evoked potentials of asphyxiated term infants. Dev Med Child Neurol 1992;34:507-15.

Thoresen M, Hellström-Westas L, Liu X, de Vries LS. Effect of hypothermia on amplitude-integrated electroencephalogram in infants with asphyxia. Pediatrics 2010;126(1):e131-9.

Toet MC, Hellström-Westas L, Groenendaal F, Eken P, de Vries LS. Amplitude integrated EEG 3 and 6 hours after birth in full term neonates with hypoxicischaemic encephalopathy. Arch Dis Child 1999;81:F19-23.

Whyte HE, Taylor MJ, Menzies R, Chin K, MacMillan L. Prognostic utility of visual evoked potentials in term asphyxiated neonates. Pediatr Neurol 1986;2 (4):220-3. 